\title{
Graded pneumatic dilation in subtype I and II achalasia: Iong-term experience in a single center
}

\section{Francesco Torresan a, Fabio Cortellini ${ }^{\mathrm{a}, \mathrm{b}}$, Francesco Azzarolia,b, Alexandros Ioannou c, Cecilia Mularoni ${ }^{\mathrm{b}}$, Dikla Shoshan ${ }^{b}$, Daniele Mandolesia, Roberto De Giorgiod, George Karamanolisc, Franco Bazzolia,b}

Azienda Ospedaliero-Universitaria di Bologna, Italy; University of Bologna, Italy; Aretaieio University Hospital, School of Medicine, National and Kapodistrian University of Athens, Greece; University of Ferrara, Italy

\section{Abstract}

${ }^{a}$ Azienda Ospedaliero-Universitaria di Bologna, Bologna, Italy (Francesco Torresan, Fabio Cortellini, Francesco Azzaroli, Daniele Mandolesi, Franco Bazzoli); ${ }^{b}$ Department of Medical and Surgical Sciences, University of Bologna, Bologna, Italy (Fabio Cortellini, Francesco Azzaroli, Cecilia Mularoni, Dikla Shoshan, Franco Bazzoli); $2^{\text {nd }}$ Department of Surgery, Gastroenterology Unit, "Aretaieio"

University Hospital, School of Medicine, National and Kapodistrian University of Athens, Athens, Greece (Alexandros Ioannou, George Karamanolis); dDepartment of Translational Medicine, University of Ferrara, Ferrara, Italy (Roberto De Giorgio)

Conflict of Interest: None

Correspondence to: Francesco Torresan, Via Massarenti 9, Bologna, Italy, e-mail: francesco.torresan@aosp.bo.it

Received 11 July 2021; accepted 25 October 2021; published online 9 December 2021

DOI: https://doi.org/10.20524/aog.2021.0683

\section{Introduction}

Achalasia is a rare esophageal motor disorder characterized by the absence of peristalsis and a defective relaxation of the lower esophageal sphincter (LES), resulting in chronic dysphagia, regurgitation and chest pain [1]. Since the introduction of high-resolution manometry (HRM) in 2008, achalasia has been classified into clinically relevant subtypes $[2,3]$. The updated Chicago classification (version 4.0) categorizes achalasia into 3 subtypes [4] that have important implications for management outcomes [5]. Despite the important step forward in diagnostic accuracy, the pathophysiology of primary achalasia remains unclear and therapeutic options are still symptomatic. Indeed, current treatment in achalasia aims to relieve symptoms by reducing the LES resting pressure [1] via 3 durable techniques used as first-line options: pneumatic dilation (PD); 
laparoscopic Heller's myotomy (LHM); and peroral endoscopic myotomy (POEM) [5]. PD is an endoscopic method that forces the hypertonic LES by intraluminal dilation of a pressurized noncompliant balloon [3]. The available evidence about the technique in the literature indicates a variable efficacy in relieving symptoms of achalasia, with success rates ranging from $50-93 \%$. These percentages, however, depend on the varied treatment protocols used and are derived from studies that do not consider the achalasia subtypes according to the Chicago classification. A limitation commonly detectable in some studies is the inclusion of subtype III patients [6-8], who manifest a poor outcome with PD [9]. To our knowledge, data on the effectiveness of PD in patients with subtypes I and II achalasia are limited.

Major complications of PD include esophageal perforation (2.3-3.5\%) and bleeding, both requiring surgery (1-4\%) [10]. In the long-term follow up, one of the main side effects is symptomatic gastroesophageal reflux disease (GERD), which occurs frequently in PD-treated patients (10-31\%) [11]. In our tertiary referral center, we used PD with a "graded approach" [12] to reduce complications and excluded subtype III achalasia patients from the analysis based on the pathophysiological concept that spastic contractions are unlikely to respond to PD. In this study we performed a retrospective analysis of the short- and long-term outcomes, safety profile and side effects of graded-protocol PD in patients with subtype I and II achalasia.

\section{Patients and methods}

This was a retrospective, observational study carried out in a tertiary referral Gastroenterology Unit at St. Orsola University Hospital, Bologna, Italy. The study protocol was approved by the Local Medical Ethical Committee (approval code: 109/2020/Oss/AOUBo).

From January 2010 to July 2020, 141 patients with achalasia were divided into 3 subtypes according to the Chicago classification (version 4.0). Although HRM was used, as it was readily available from 2015 on, previous diagnoses of achalasia were established via conventional manometry and then re-categorized into the 3 subtypes as described by Salvador et al [13] Accordingly, one of the authors (FT) reevaluated the full digital esophageal tracings obtained with conventional manometry using the electric pump software (Mui Scientific, Mississauga, Canada). We considered the contraction waves recorded 5 and $10 \mathrm{~cm}$ above the upper margin of the LES. Patients were classified into the achalasia subtypes as follows: subtype I, at least 8 of 10 swallows elicited contractions with an amplitude $<30 \mathrm{mmHg}$; subtype II, 2 or more contractions with an amplitude $>30 \mathrm{mmHg}$; subtype III, at least 2 spastic contractions with an amplitude $>70 \mathrm{mmHg}$ and duration $>6.0 \mathrm{sec}$.

Inclusion criteria were as follows: patients aged $>18$ years with achalasia subtype I or II referred for esophageal manometry. Patients with subtype III achalasia $(n=40)$ were evaluated exclusively for epidemiology. On the other hand, patients who had undergone previous treatments (i.e., previous PD, LHM or POEM) or were of advanced age $(>75$ years $)$ and/or severe comorbidities $(n=25)$, were managed with safer treatments, such as botulinum toxin injections [14], and were therefore excluded from this study. Secondary forms of achalasia, resulting from esophageal cancer or other infiltrative diseases, were ruled out via upper gastrointestinal endoscopy.

\section{PD procedure}

Endoscopic PD was performed by a single, experienced operator (FT) using a 30, 35 or $40 \mathrm{~mm}$ diameter dilation balloon (Rigiflex ABD ${ }^{\oplus}$, Boston Scientific, Natick, USA). The balloon was placed over an endoscopically introduced guide wire and positioned across the LES under fluoroscopic guidance. Once in place, the balloon was left inflated at 1015 pounds per square inch (psi) for $60 \mathrm{sec}$. The procedures were carried out on an inpatient basis until 2016, and from that year on in outpatients discharged after 4-6 h postdilation following a contrast (gastrografin) esophagram to rule out perforations. In order to minimize undesirable events, we used a "graded approach": i.e., starting with a 30-mm diameter balloon and progressing, if necessary, to a larger diameter balloon. The need for further dilation was determined by the persistence of symptoms (Eckardt score >3) 4 weeks after the procedure. Patients with persistence of symptoms underwent an elective additional dilation with $35 \mathrm{~mm}$ diameter balloon. A third dilation with 40$\mathrm{mm}$ diameter balloon was performed in those unresponsive to the second dilation. Patients with Eckardt scores $>3$ at 4 weeks after the third dilation were considered early failures and referred to other treatments. Patients achieving an Eckardt score $\leq 3$ at the end of the first cycle of the graded approach were considered as successful treatments in the short-term outcome. To assess long-term outcome, we conducted annual follow-up evaluations with Eckardt score. Four patients died for other reasons during the follow up ( 2 from cardiac failure, 1 from a cerebrovascular event, and 1 from sepsis). Additional redilation series were proposed to those who showed clinical relapses during follow up, starting with the last balloon diameter used. The incidence of GERD following the procedure was determined based on the new-onset use of proton pump inhibitors. Median follow up was 56 months (range 2-137) for both types, i.e., 89 months (24-137) for subtype I, and 38 months (2-131) for subtype II.

\section{Statistical analysis}

Descriptive analysis was carried out using the appropriate statistics, such as mean, median, standard deviation and confidence intervals. The D'Agostino-Pearson test for normal distribution was applied for the baseline characteristics variables. We presented the epidemiological data for the entire 
population included in the study. To assess the differences before and after therapy we used the Student's $t$-test for continuous variables and the $\chi^{2}$ test for categorical variables. Treatment success was defined as an Eckardt score $\leq 3$. To evaluate which variables were associated with therapeutic success, we carried out a Cox logistic regression analysis. All P-values were 2-tailed and a P-value $<0.05$ was considered statistically significant. Data were analyzed using MedCalc 19.6.4 (MedCalc Software Ltd, Ostend, Belgium).

\section{Results}

A final cohort of 76 patients met the inclusion and exclusion criteria (Fig. 1). Of these, 4 patients were lost to follow up and were not analyzed for the long-term results. The baseline characteristics of the study population are summarized in Table 1. The mean pre-dilation Eckardt score was $7.2 \pm 1.86$ in the subtype I group and $7.05 \pm 1.89$ in subtype II.

Table 1 Baseline characteristics of the retrospective cohort

\begin{tabular}{lcccc}
\hline Characteristics & Subtype I & Subtype II & Total & P-value \\
\hline $\mathrm{n}(\%)$ & 27 & 74 & 101 & \\
Age & $67 \pm 17.63$ & $62 \pm 14.23$ & $66 \pm 16.26$ & 0.001 \\
Female & $12(45 \%)$ & $40(54 \%)$ & $52(51 \%)$ & - \\
Male & $15(55 \%)$ & $34(46 \%)$ & $49(49 \%)$ & - \\
$\begin{array}{l}\text { Eckardt score } \\
\text { pre-dilation }\end{array}$ & $\begin{array}{c}7.2 \pm 1.86 \\
(\mathrm{n}=24)\end{array}$ & $\begin{array}{c}7.05 \pm 1.89 \\
(\mathrm{n}=52)\end{array}$ & $\begin{array}{c}7.13 \pm 2 \\
(\mathrm{n}=76)\end{array}$ & - \\
\hline
\end{tabular}

\section{Short-term outcome}

Among the 24 subtype I patients, 23 (95.8\%) reached clinical remission (defined as Eckardt score $\leq 3$ ) after the first PD. One subject achieved remission after the second dilation, leading to a $100 \%$ success rate in the first series of PDs. The $40 \mathrm{~mm}$ diameter balloon was not necessary in subtype I achalasia. In the 52 patients with subtype II, the first dilation was effective in $40(76.9 \%)$, the second in $8(92.3 \%)$, and the third in 2 cases (96.2\%). Only one patient from the subtype II group refused permission to continue the graded approach after a first unsuccessful PD. At the end of the graded protocol, 74 of the 76 patients from both groups had successful treatment (success rate $97.4 \%$; Table 2). A total of 90 PDs were completed without major complications (i.e., perforation and bleeding requiring surgery): 25 for subtype I (average/patient=1.04) and 65 for subtype II (average/patient=1.25).

\section{Long-term outcome}

The long-term outcome after a median period of 56 months was as follows: $90.9 \%$ subtype I and $86 \%$ subtype II achalasia patients were in clinical remission (Table 3). In the subtype I group, among the 22 patients who responded to the first series of PDs and completed the follow up, 2 patients (9.1\%) showed clinical recurrence and so underwent a redilation starting from the balloon diameter used in the last procedure. These 2 patients were treated with a $35 \mathrm{~mm}$ balloon: 1 achieved clinical remission, whereas the other did not. Indeed, the latter patient failed even after the $40 \mathrm{~mm}$ diameter balloon dilation

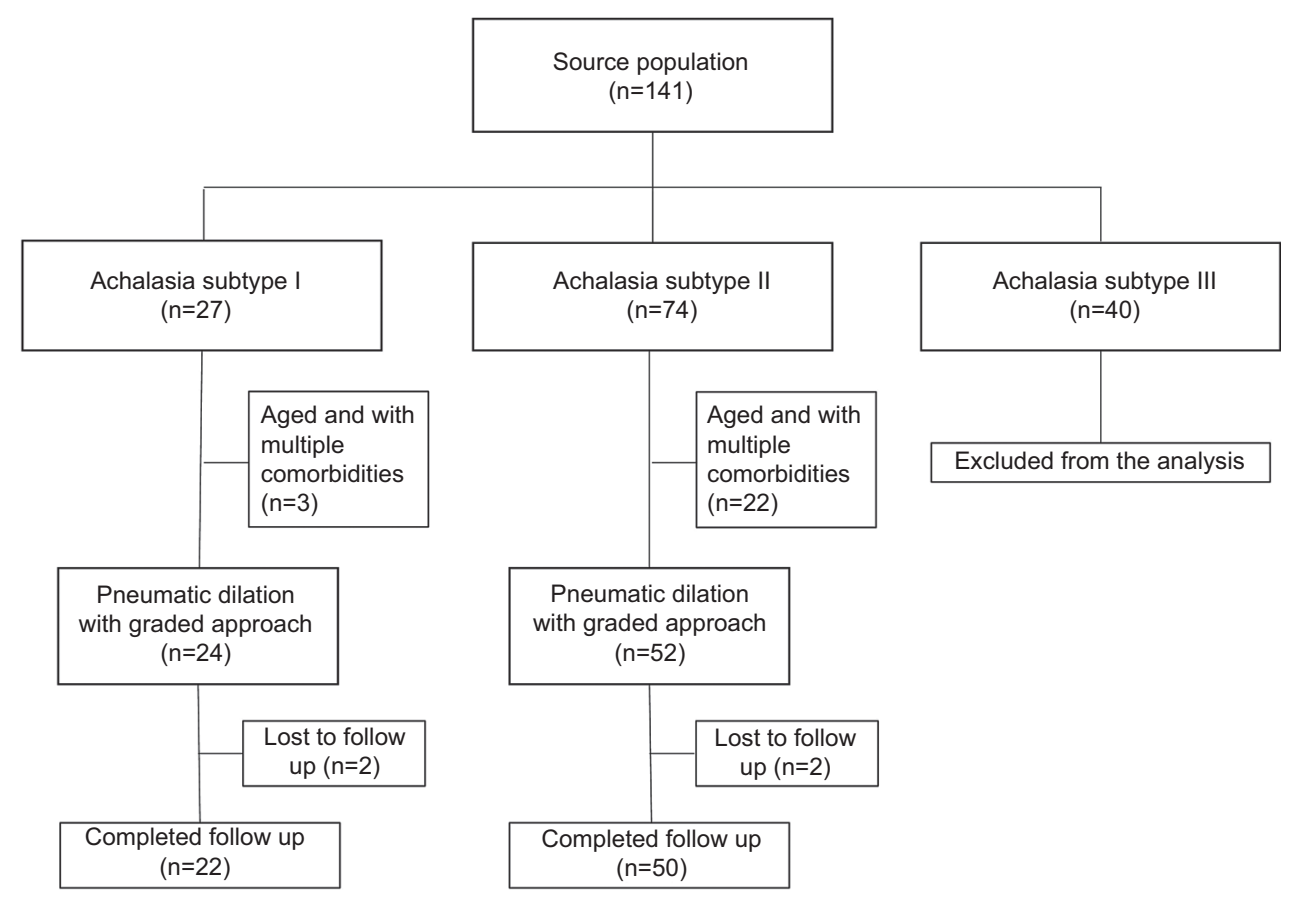

Figure 1 Study flowchart 
Table 2 Short-term results and complications of pneumatic dilation in achalasia subtype I and II

Achalasia subtype I

\begin{tabular}{lcccccc}
\hline Dilation & $\mathrm{N}$ & $\begin{array}{c}\text { Eckardt score } \\
\text { (pre-dilation) }\end{array}$ & $\begin{array}{c}\text { Eckardt score } \\
\text { (post-dilation) }\end{array}$ & P-value & $\begin{array}{c}\text { Eckardt score<3 } \\
\text { after 1 month }(\%)\end{array}$ & $\begin{array}{c}\text { Complication rate } \\
(\%)\end{array}$ \\
\hline 1st dilation $(30 \mathrm{~mm})$ & 24 & $7.2 \pm 1.86$ & $1.4 \pm 1.92$ & $<0.001$ & $23(95.8 \%)$ & 0 \\
2nd dilation $(35 \mathrm{~mm})$ & 1 & 11 & 2 & - & $1(4.2 \%)$ & - \\
3rd dilation $(40 \mathrm{~mm})$ & 0 & - & - & - & - & 0 \\
$\begin{array}{l}\text { Final results at the end of the } \\
\text { graded protocol }\end{array}$ & 24 & $7.2 \pm 1.86$ & $1.4 \pm 1.89$ & $<0.001$ & $24(100 \%)$ & \\
\hline
\end{tabular}

Achalasia subtype II

\begin{tabular}{|c|c|c|c|c|c|c|}
\hline Dilation & $\mathrm{N}$ & $\begin{array}{l}\text { Eckardt score } \\
\text { (pre-dilation) }\end{array}$ & $\begin{array}{l}\text { Eckardt score } \\
\text { (post-dilation) }\end{array}$ & P-value & $\begin{array}{l}\text { Eckardt score }<3 \\
\text { after } 1 \text { month }(\%)\end{array}$ & $\begin{array}{l}\text { Complication rate } \\
(\%)\end{array}$ \\
\hline 1st dilation $(30 \mathrm{~mm})$ & 52 & $7.05 \pm 1.89$ & $1.76 \pm 2.09$ & $<0.001$ & $40(76.9 \%)$ & 0 \\
\hline 2nd dilation (35 mm) & 11 & $6.8 \pm 1.89$ & $2.9 \pm 1.97$ & $<0.001$ & $8(15.4 \%)$ & 0 \\
\hline 3rd dilation $(40 \mathrm{~mm})$ & 2 & $6 \pm 1$ & $1 \pm 1$ & - & $2(3.8 \%)$ & 0 \\
\hline $\begin{array}{l}\text { Final results at the end of the } \\
\text { graded protocol }\end{array}$ & 52 & $7.05 \pm 1.89$ & $1.26 \pm 1.48$ & $<0.001$ & $50(96.2 \%)$ & 0 \\
\hline & \multicolumn{6}{|c|}{ Both subtypes } \\
\hline $\begin{array}{l}\text { Final results at the end of the } \\
\text { graded protocol for both subtypes }\end{array}$ & 76 & $7.13 \pm 1.87$ & $1.17 \pm 1.36$ & $<0.01$ & $74(97.4 \%)$ & 0 \\
\hline
\end{tabular}

Table 3 Long-term results and side-effects of pneumatic dilation in achalasia subtype I and II

\begin{tabular}{lccccccc}
\hline Achalasia subtype & $\mathrm{N}$ & $\begin{array}{c}\text { Follow up } \\
\text { (median) }\end{array}$ & $\begin{array}{c}\text { Clinical remission } \\
\text { at follow up (\%) }\end{array}$ & $\begin{array}{c}\text { Remission } \\
\text { after redilation }\end{array}$ & $\begin{array}{c}\text { Eckardt score } \\
\text { at follow up }\end{array}$ & P-value & GERD \\
\hline Achalasia subtype I & 22 & $89(24-137)$ & $20(90.9 \%)$ & $21(95.5 \%)$ & $1.27 \pm 1.12$ & $<0.001$ & $6(27.3 \%)$ \\
Achalasia subtype II & 50 & $38(2-131)$ & $43(86 \%)$ & $45(90 \%)$ & $1.96 \pm 2.04$ & $<0.001$ & $14(28 \%)$ \\
Total & 72 & $56(2-137)$ & $63(87.5 \%)$ & $65(90.3 \%)$ & $1.75 \pm 1.82$ & $<0.001$ & 20 \\
& & & & & & $(27.8 \%)$ \\
\hline
\end{tabular}

GERD, gastroesophageal reflux disease

(Eckardt score at 4 weeks=5) and was therefore referred for surgery. Considering redilations, the long-term success of PD in our series reached $95.5 \%$ in subtype I achalasia.

In the subtype II group, 7 of 50 patients (14\%) presented with a relapse during the follow up. Four had undergone only the first dilation, one of them had one dilation with a $35-\mathrm{mm}$ diameter balloon and 2 patients completed the graded protocol with the last $40 \mathrm{~mm}$ diameter balloon dilation. Two patients underwent a further procedure with a $35-\mathrm{mm}$ balloon, leading to clinical remission. The remaining 5 did not benefit from redilations and were referred for different treatments, either LHM or POEM. A "re-do" strategy, with additional dilations during the follow up, permitted a clinical remission rate of $90 \%$. None of the patients who underwent redilations had major complications, while none of the variables analyzed (age, sex, achalasia subtype, Eckardt score before treatment, time interval between diagnosis and treatment) showed any correlation with therapeutic success. Finally, we evaluated the incidence of postoperative GERD: $27.8 \%$ of the patients treated with PD needed proton pump inhibitors after the treatment.

\section{Discussion}

PD was the first endoscopic technique used to treat achalasia symptoms, regardless of the underlying manometric pattern, until POEM was developed [15]. By increasing the pressure inside the inflatable balloon, the therapeutic action of PD aims to stretch the smooth muscle fibers of the LES, thereby achieving an effective dilation of the sphincter. Thus, unlike other techniques such as POEM, it cannot act on the symptoms that result from contraction and pressurization of the proximal muscle of the esophagus, such as patients with a specific subset of achalasia characterized by prominent contractions (formerly referred to as "vigorous achalasia") [16,17]. This observation became more apparent with the advent of HRM and pressure topography plotting, which precisely delineated 
the manometric characteristics of these patients and defined them as subtype III achalasia [18]. Comparison studies have confirmed a poorer response of subtype III to PD compared with POEM, which allows smooth muscle dissection up to proximal esophageal segments [19-21]. For this reason, we opted to include in our cohort only subtype I and II patients treated with PD.

The results reported in this retrospective study, from a cohort of patients homogeneous as regards sex and age, showed excellent short-term success rates of $100 \%$ and $96.2 \%$ for subtypes I and II achalasia, respectively. Because of the chronic nature of this esophageal disorder, we also analyzed long-term outcomes with later redilations, reaching clinical remission in $95.5 \%$ and $90 \%$ of subtype I and II patients over a median follow up of 7 and 3 years, respectively. Our study did not show any correlation between male sex and worse outcome, as previously suggested by some authors [22,23]. In fact, a large meta-analysis that investigated patient-specific predictors has recently reported a lack of any association between sex and treatment outcome [24].

Most of the data on the efficacy of PD in the treatment of achalasia include subgroup III, and thus yield a lower response rate than those reported in this study, where we have selected only subtypes I and II [25-30]. Recently, a meta-analysis by Andolfi et al reported the success rates of PD for each achalasia subtype: $61 \%$ and $84 \%$ in subtypes I and II, respectively, after a mean follow up of 24 months. Comparing PD with other treatments, such as LHM and POEM, the efficacy was clearly in favor of these 2 approaches, yielding respective success rates of $81 \%$ and $95 \%$ in subtype I and $92 \%$ and $97 \%$ in subtype II [9]. Moreover, a prospective clinical trial showed a poor efficacy for PD compared with excellent results from POEM (53\% vs. $92 \%$ after 2 years) [21]. These results, however, are based on studies that used different dilation protocols and definitions of clinical success [12]. These criticisms can be extended to the majority of meta-analyses comparing distinct treatment methods in achalasia, thereby leading experts to conclude that with the currently available data it is difficult to establish which treatment option is the best for any single patient [31]. Notably, our results were in line with the excellent success rates showed by Rohof et al, who used a protocol of graded dilation with redilations in patients with symptom recurrence [32].

The graded PD protocol that we have been using since 2010 is the safest and most effective one among those available [12], and was recently recommended by European Society of Gastrointestinal Endoscopy guidelines as a therapeutic option [33]. The absence of complications in the short term demonstrated in our study confirms and expands previous evidence for PD safety. A gradual dilation starting from a $30-\mathrm{mm}$ balloon aims to adapt the diameter of the device to the esophageal caliber, to allow progressive and safe stretching of the smooth muscle fibers. On the other hand, in the long-term, the occurrence of reflux was considerable, i.e., $22.3 \%$ and $28 \%$ in subtypes I and II, respectively, although these results were consistent with published data [11]. All the procedures were performed in a tertiary referral center for esophageal dysmotility and thoracic surgery was available as a back-up in case of complications. Initially, we applied the procedure to inpatients, whereas more recently we opted for an outpatient setting, with the advantage of greater patient compliance and lower costs for the hospital. Although this is not recommended by guidelines [34], patients who underwent PD were checked with a contrast (gastrografin) esophagogram before being discharged.

This study clearly had several limitations that should be acknowledged. First, its retrospective design is known to hamper the power of the obtained results and is open to possible biases. Secondly, a considerable number of patients, mostly subtype II achalasia, had to be excluded from PD treatment because of advanced age and/or severe comorbidities.

In conclusion, according to our study, PD is still a safe and effective approach for treating patients with achalasia subtype I and II. Notably, PD can be performed in an outpatient setting with reasonable costs, provided that the procedure is performed in a tertiary referral center with experienced operators and thoracic surgery available to manage possible complications promptly. The other 2 important messages that we would like to convey are: 1) the efficacy of PD in achalasia should always consider the achalasia subtype (with subtypes I and II being most suitable for PD treatment); and 2) the PD procedure should be performed using a graded approach, as applied in this study and recommended by most important pertinent papers on this topic.

\section{Acknowledgment}

The authors would like to thank Mr. Cade Hannan for language and style corrections

\section{Summary Box}

\section{What is already known:}

- Achalasia is a rare esophageal motor disorder with unclear pathophysiology and only symptomatic treatment is available

- Pneumatic dilation shows a variable efficacy in relieving achalasia symptoms

- Current data on pneumatic dilation present heterogeneous dilation protocols and include subtype III achalasia

\section{What the new findings are:}

- In an Italian retrospective cohort of patients with achalasia subtype I and II, pneumatic dilation with a graded protocol achieved excellent short-term outcomes

- Repeated dilations during follow up ensured an optimal outcome in the long-term

- The graded approach, performed in a tertiary referral center with thoracic surgery, can be used safely in an outpatient setting 


\section{References}

1. Pandolfino JE, Gawron AJ. Achalasia: a systematic review. JAMA 2015;313:1841-1852.

2. Pandolfino JE, Kwiatek MA, Nealis T, Bulsiewicz W, Post J, Kahrilas PJ. Achalasia: a new clinically relevant classification by high-resolution manometry. Gastroenterology 2008;135:1526-1533.

3. Torresan F, Ioannou A, Azzaroli F, Bazzoli F. Treatment of achalasia in the era of high-resolution manometry. Ann Gastroenterol 2015;28:301-308.

4. Yadlapati R, Kahrilas PJ, Fox MR, et al. Esophageal motility disorders on high-resolution manometry: Chicago classification version 4.0(@). Neurogastroenterol Motil 2021;33:e14058.

5. Kahrilas PJ, Pandolfino JE. Treatments for achalasia in 2017: how to choose among them. Curr Opin Gastroenterol 2017;33:270-276.

6. Vaezi MF, Richter JE. Diagnosis and management of achalasia. American College of Gastroenterology Practice Parameter Committee. Am J Gastroenterol 1999;94:3406-3412.

7. Vela MF, Richter JE, Khandwala F, et al. The long-term efficacy of pneumatic dilatation and Heller myotomy for the treatment of achalasia. Clin Gastroenterol Hepatol 2006;4:580-587.

8. Campos GM, Vittinghoff E, Rabl C, et al. Endoscopic and surgical treatments for achalasia: a systematic review and meta-analysis. Ann Surg 2009;249:45-57.

9. Andolfi C, Fisichella PM. Meta-analysis of clinical outcome after treatment for achalasia based on manometric subtypes. Br J Surg 2019;106:332-341.

10. Khashab MA, Vela MF, Thosani N, et al. ASGE guideline on the management of achalasia. Gastrointest Endosc 2020;91:213-227.

11. Oude Nijhuis RAB, Zaninotto G, Roman S, et al. European Guideline on Achalasia - UEG and ESNM recommendations. United European Gastroenterol J 2020;8:13-34.

12. van Hoeij FB, Prins LI, Smout AJPM, Bredenoord AJ. Efficacy and safety of pneumatic dilation in achalasia: a systematic review and meta-analysis. Neurogastroenterol Motil 2019;31: e13548.

13. Salvador R, Costantini M, Zaninotto G, et al. The preoperative manometric pattern predicts the outcome of surgical treatment for esophageal achalasia. J Gastrointest Surg 2010;14:1635-1645.

14. Gui D, Rossi S, Runfola M, Magalini SC. Review article: botulinum toxin in the therapy of gastrointestinal motility disorders. Aliment Pharmacol Ther 2003;18:1-16.

15. Phalanusitthepha $\mathrm{C}$, Inoue $\mathrm{H}$, Ikeda $\mathrm{H}$, et al. Peroral endoscopic myotomy for esophageal achalasia. Ann Transl Med 2014;2:31.

16. Khashab MA, Messallam AA, Onimaru M, et al. International multicenter experience with peroral endoscopic myotomy for the treatment of spastic esophageal disorders refractory to medical therapy (with video). Gastrointest Endosc 2015;81:1170-1177.

17. Khan MA, Kumbhari V, Ngamruengphong S, et al. Is POEM the answer for management of spastic esophageal disorders? A systematic review and meta-analysis. Dig Dis Sci 2017;62:35-44.

18. Gregersen H, Lo KM. Pathophysiology and treatment of achalasia in a muscle mechanical perspective. Ann N Y Acad Sci 2018;1434:173-184.

19. Jung HK, Hong SJ, Lee OY, et al; Korean Society of Neurogastroenterology and Motility. 2019 Seoul consensus on esophageal achalasia guidelines. J Neurogastroenterol Motil 2020;26:180-203.

20. Kumbhari V, Tieu AH, Onimaru M, et al. Peroral endoscopic myotomy (POEM) vs laparoscopic Heller myotomy (LHM) for the treatment of Type III achalasia in 75 patients: a multicenter comparative study. Endosc Int Open 2015;3:E195-E201.

21. Ponds FA, Fockens P, Lei A, et al. Effect of peroral endoscopic myotomy vs pneumatic dilation on symptom severity and treatment outcomes among treatment-naive patients with achalasia: a randomized clinical trial. JAMA 2019;322:134-144.

22. Ghoshal UC, Kumar S, Saraswat VA, Aggarwal R, Misra A, Choudhuri G. Long-term follow-up after pneumatic dilation for achalasia cardia: factors associated with treatment failure and recurrence. Am J Gastroenterol 2004;99:2304-2310.

23. Farhoomand K, Connor JT, Richter JE, Achkar E, Vaezi MF. Predictors of outcome of pneumatic dilation in achalasia. Clin Gastroenterol Hepatol 2004;2:389-394.

24. Oude Nijhuis RAB, Prins LI, Mostafavi N, van Etten-Jamaludin FS, Smout AJPM, Bredenoord AJ. Factors associated with achalasia treatment outcomes: systematic review and meta-analysis. Clin Gastroenterol Hepatol 2020;18:1442-1453.

25. Pratap N, Kalapala R, Darisetty S, et al. Achalasia cardia subtyping by high-resolution manometry predicts the therapeutic outcome of pneumatic balloon dilatation. J Neurogastroenterol Motil 2011;17:48-53.

26. Min M, Peng LH, Yang YS, et al. Characteristics of achalasia subtypes in untreated Chinese patients: a high-resolution manometry study. J Dig Dis 2012;13:504-509.

27. Yamashita H, Ashida K, Fukuchi T, et al. Predictive factors associated with the success of pneumatic dilatation in japanese patients with primary achalasia: a study using high-resolution manometry. Digestion 2013;87:23-28.

28. Park JH, Lee YC, Lee H, et al. Residual lower esophageal sphincter pressure as a prognostic factor in the pneumatic balloon treatment of achalasia. J Gastroenterol Hepatol 2015;30:59-63.

29. Meng F, Li P, Wang Y, et al. Peroral endoscopic myotomy compared with pneumatic dilation for newly diagnosed achalasia. Surg Endosc 2017;31:4665-4672.

30. Müller M, Keck C, Eckardt AJ, et al. Outcomes of pneumatic dilation in achalasia: Extended follow-up of more than 25 years with a focus on manometric subtypes. $J$ Gastroenterol Hepatol 2018;33:1067-1074.

31. de Heer J, Desai M, Boeckxstaens G, et al. Pneumatic balloon dilatation versus laparoscopic Heller myotomy for achalasia: a failed attempt at meta-analysis. Surg Endosc 2021;35:602-611.

32. Rohof WO, Salvador R, Annese V, et al. Outcomes of treatment for achalasia depend on manometric subtype. Gastroenterology 2013;144:718-725.

33. Weusten BLAM, Barret M, Bredenoord AJ, et al. Endoscopic management of gastrointestinal motility disorders - part 1: European Society of Gastrointestinal Endoscopy (ESGE) Guideline. Endoscopy 2020;52:498-515.

34. Vaezi MF, Pandolfino JE, Yadlapati RH, Greer KB, Kavitt RT. ACG Clinical Guidelines: diagnosis and management of achalasia. Am J Gastroenterol 2020;115:1393-1411. 